\title{
RECONSTRUÇÃO ESOFÁGICA PELA ESOFAGOCOLOPLASTIA NO MEGAESÔFAGO AVANÇADO EM PACIENTES GASTRECTOMIZADOS
}

\section{Esophageal reconstruction by esophagocoloplasty for megaesophagus end-stage in gastrectomized patients}

\author{
Jose Luis Braga de AQUINO, Marcelo Manzano SAID, Diana MAZIERO, \\ Jean Marc Prado de OLIVEIRA, Vania Aparecida LEANDRO-MERHI
}

ABCDDV/672

Aquino JLB, Said MM, Maziero D, Pliveira JMP, Leando-Merhi VA. Reconstrução esofágica pela esofagocoloplastia no megaesôfago avançado em pacientes gastrectomizados. ABCD Arq Bras Cir Dig 2009;22(4):197-200

RESUMO - Racional - A necessidade de esofagocoloplastia em pacientes com megaesôfago avançado, previamente submetidos à gastrectomia não é frequente, mas quando executada traz consigo maior dificuldade técnica, o que pode elevar o número de complicações. Objetivo - Avaliar as complicações da reconstrução de trânsito pela esofagocoloplastia em uma série de pacientes submetidos à esofagectomia transmediastinal por megaesôfago avançado com gastrectomia prévia. Métodos - De julho de 1983 a abril de 2009, 204 pacientes com megaesôfago grau III e IV foram submetidos à ressecção esofágica no Departamento de Cirurgia do Hospital Celso Pierro da Puc-Campinas. Em 92 pacientes a ressecção foi pela técnica da mucosectomia esofágica; em 84 pela via transmediastinal; em 38 pela via transtorácica. Em 194 pacientes (95\%) a reconstrução do trânsito foi realizada pela transposição gástrica e nos 10 restantes $(5 \%)$, pela transposição do colo transverso. O procedimento foi indicado pelo fato dos pacientes já terem sido submetidos à gastrectomia prévia. Todos eram do sexo masculino, com idade média de 47, 5 anos. Resultados - Sete pacientes $(70 \%)$ apresentaram uma ou mais complicações, sejam sistêmicas ou locais. Das sistêmicas, um paciente apresentou tromboflebite em membro inferior, com boa evolução; outro, infarto do miocárdio, com evolução fatal; três pacientes (30\%) apresentaram infecção pulmonar, com boa evolução. Das complicações locais, quatro tiveram deiscência da anastomose esofagocolônica cervical, tendo boa evolução com tratamento conservador. Cinco pacientes evoluíram com estenose da anastomose esofagocolônica cervical entre o $35^{\circ}$ e $63^{\circ}$ dia do pós-operatório, sendo realizadas dilatações endoscópicas com boa evolução. De oito pacientes acompanhados neste período, seis $(75 \%)$ apresentavam boa deglutição para sólidos e/ou pastosos, referindo estarem satisfeitos com ato cirúrgico por terem retornado aos hábitos usuais. Conclusões - A reconstrução com o colo em pacientes submetidos à esofagectomia por megaesôfago avançado não deve ser a primeira opção de tratamento, tendo somente indicação quando for inviável a transposição gástrica, e ela deve ser considerada técnica cirúrgica com alta morbimortalidade em pacientes previamente gastrectomizados.

DESCRITORES - Reconstrução esofágica. Megaesôfago chagásico. Esofagocoloplastia.

\section{INTRODUÇÃO}

Ao longo da história cirúrgica mundial, a esofagectomia sempre proporcionou grande temor devido ao elevado potencial de complicações e, não raro mortalidade mais elevada, justificando controvérsia até os dias atuais sobre qual é o melhor tipo de ressecção esofágica e de reconstrução.

No megaesôfago avançado, este fato torna-se mais evidente por se atuar em pacientes desnutridos, muitas vezes com alterações cardiovasculares, consequentes a potencial acometimento chagásico do miocárdio, o que pode proporcionar maior morbidade no pós-operatório. Apesar disso, a esofagectomia ainda é o procedimento mais utilizado na maioria das séries para o tratamento desta afecção por se atuar diretamente na doença ao remover o órgão como um todo ${ }^{1,3,4,5,6,7,8,11,12,13,14,15,16,17}$. Autores têm enfatizado que o órgão de eleição para reconstrução de trânsito após a ressecção esofágica é o estômago, pelas vantagens que

Trabalho realizado no Serviço de Cirurgia de Tórax do Hospital Maternidade Celso Pierro da Faculdade de Medicina da Pontifícia Universidade Católica de Campinas, Campinas, SP, Brasil

Correspondência: José Luis Braga de Aquino, e-mail: jlaquino@sigmanet.com.br oferece: reconstrução mais fisiológica estabelecendo de maneira contínua e direta o trânsito digestivo; bem vascularizado proporcionando menor índice de complicações diretamente relacionadas à anastomose; realização de uma única anastomose para reconstrução do trânsito digestivo; boa mobilidade sendo transposto com mais facilidade até a região cervical; menor contaminação.

A reconstrução com o colo, por outro lado, tem sido cada vez menos utilizada pela maior morbidade, por ser procedimento cirúrgico mais complexo, com realização de várias anastomoses e maior tempo de operação.

Os poucos pacientes que ainda têm indicação de realização de esofagocoloplastia após ressecção esofágica para megaesôfago avançado seriam aqueles que já foram submetidos previamente à gastrectomia ou por condições anatômicas desfavoráveis $9,10,14,17$.

Nos últimos anos, por não haver na literatura nacional nenhum estudo que demonstrasse os resultados da esofagocoloplastia em pacientes com megaesôfago avançado previamente submetidos à gastrectomia, surgiu a idéia de demonstrar os resultados deste tipo de reconstrução.

Assim sendo, o objetivo deste estudo é analisar retrospectivamente, as complicações da utilização do colo transverso para reconstrução do trato digestivo superior 
após a ressecção do esôfago em uma série de pacientes com megaesôfago avançado anteriormente submetidos à gastrectomia por doença benigna.

\section{MÉTODOS}

De julho de 1983 a abril de 2009, 204 pacientes com megaesôfago grau III e IV foram submetidos à ressecção esofágica no Departamento de Cirurgia do Hospital Celso Pierro da Puc-Campinas. Em 92 pacientes a ressecção foi pela técnica da mucosectomia esofágica (Aquino, 1991,1996), em 84 pela via transmediastinal (Pinotti, 1976) e em 38 pela via transtorácica (Câmara Lopes \& Ferreira Santos, 1955, 1958). Em 194 pacientes (95\%) a reconstrução do trânsito foi realizada pela transposição gástrica e nos 10 restantes $(5 \%)$, pela transposição do colo transverso que é o objeto deste estudo. Todos pacientes apresentavam diagnóstico sorológico por imunofluorescência direta para doença de Chagas positivo.

A transposição colônica foi indicada pelo fato dos pacientes já terem sido submetidos à gastrectomia prévia por úlcera péptica entre 11 e 28 anos. Destes, oito tiveram reconstrução à Billroth II e dois, à Billroth I. Todos os pacientes eram do sexo masculino com idade variável entre 39 e 68 anos, com média de 47, 5 anos.

Nos 10 pacientes, a ressecção esofágica foi por via transmediastinal com transposição do colo transverso pela via mediastinal posterior. Foi realizada anastomose esofagocolônica a nível cervical, com sutura manual, em dois planos e em posição término-terminal; anastomose cologástrica em oito pacientes e nos dois restantes, anastomose colojejunal; em todos foi realizada reconstrução do trânsito colônico pela anastomose do colo descendente com colo ascendente em posição término-términal. Em todos foi realizada jejunostomia para suporte nutricional no pós-operatório até a liberação da dieta via oral, a partir do sétimo dia de pós-operatório, desde que o paciente não tivesse evidência clínica ou radiológica de fístula. Todos os pacientes foram submetidos à drenagem de tórax bilateralmente. Todas as operações foram realizadas pelo mesmo cirurgião, com a mesma equipe.

\section{RESULTADOS}

Sete pacientes $(70 \%)$ apresentaram uma ou mais complicações, sejam sistêmicas ou locais. Das sistêmicas, um apresentou tromboflebite em membro inferior, com boa evolução por tratamento específico; e outro apresentou infarto do miocárdio, com evolução fatal no $6^{\circ}$ dia do pósoperatório; três $(30 \%)$ apresentaram infecção pulmonar, associado ou não a derrame pleural com boa evolução com tratamento clínico. No que concerne às complicações locais, quatro tiveram deiscência da anastomose esofagocolônica cervical, tendo boa evolução com tratamento conservador, e fechamento da fístula em tempo que variou de 20 a 30 dias. Após este período, foi realizado estudo radiográfico contrastado e, na ausência de qualquer sinal de extravasamento de contraste, introduziu-se dieta via oral. Os quatro que tiveram deiscência da anastomose, e outro que não apresentou esta complicação, evoluíram com estenose da anastomose esofagocolônica cervical entre o $35^{\circ}$ e $63^{\circ}$ dia do pós-operatório. Como tratamento, foram realizadas dilatações endoscópicas, em caráter ambulatorial, com boa evolução clínica. Um paciente apresentou deiscência da anastomose cologástrica, tendo sido submetido à reintervenção cirúrgica no $7^{\circ}$ dia do pós-operatório, para drenagem do abscesso intraperitoneal, com boa evolução. Este paciente pertenceu ao grupo dos que apresentaram deiscência da anastomose esofagocolônica cervical.

De oito pacientes acompanhados de 2 a 13 anos, seis (75\%) apresentavam boa deglutição para sólidos e/ou pastosos, referindo estarem satisfeitos com ato cirúrgico por terem retornado aos hábitos usuais.

\section{DISCUSSÃO}

Apesar da reconstrução com o colo após a ressecção esofágica por megaesôfago avançado já ter sido preconizada há muitos anos por Rassi ${ }^{18,19,20,21}$, este tipo de reconstrução, nos últimos anos, tem tido pouca indicação, geralmente não ultrapassando a $10 \%$ na maioria das séries estudadas ${ }^{6,8,11,12,14,17,23}$. E isto também ficou demonstrado nesta casuística, pois de 204 pacientes submetidos à esofagectomia para megaesôfago avançado, a indicação da esofagocoloplastia ocorreu apenas em 5\%, resumindo-se apenas aos 10 pacientes estudados nesta série; assim, a reconstrução de eleição foi com o estômago na maioria dos pacientes ${ }^{2}$. A indicação para realização da transposição colônica deu-se exclusivamente devido aos pacientes serem gastrectomizados previamente por doença benigna, o que inviabilizaria qualquer tentativa de transposição gástrica transmediastinal para reconstrução do trânsito digestivo. Este fato tem sido corroborado por outros autores que também contraindicam a transposição colônica ${ }^{1,3,6,13,14}$. Os que contraindicam - onde os autores desta série se incluem -, o fazem pela potencial degeneração associada que o colo pode apresentar, o que poderia comprometer a deglutição pela diminuição da contratilidade desta víscera ao ser transposta até a região cervical ${ }^{1,3,7,14}$. Entretanto, nos oito pacientes acompanhados a curto e médio prazo, $75 \%$ referiram estarem satisfeitos com o ato cirúrgico, com boa deglutição para sólidos ou pastosos. Estes bons resultados talvez tenham ocorrido devido aos pacientes terem sido avaliados por pouco tempo, o que, possivelmente, não conseguiu demonstrar as alterações da deglutição pelo potencial comprometimento neuronal do colo interposto, gerando, principalmente, a estase de alimentos. Daí a importância de se realizar um acompanhamento a longo prazo com maior número de pacientes

Outro óbice para indicação da esofagocoloplastia após esofagectomia para megaesôfago avançado é o fato de o colo ser menos vascularizado que o estômago, podendo assim aumentar a incidência e a intensidade da deiscência das anastomoses. Isto se refletiu nesta casuística, pois $40 \%$ dos pacientes apresentaram deiscência da anastomose cervical. Apesar de apresentarem boa evolução com 
tratamento conservador, houve retardo para início da deglutição de 20 a 30 dias. Além disso, um destes pacientes com esta complicação também apresentou deiscência na anastomose cologástrica, tendo necessidade de reoperação, o que aumentou a morbidade pós-operatória. Embora com menor incidência que nesta série, esta complicação também tem sido demonstrada por outros que utilizam o colo para reconstrução do trânsito nestas situações ${ }^{6,12,13,14}$. Talvez se tivesse sido utilizada a sutura mecânica circular que, por ser dupla, invertida e estimular maior neoangiogênese, poder-se-ia minimizar esta complicação ao nível da anastomose cervical, como tem sido demonstrado nos últimos anos em várias séries que utilizam este tipo de sutura para reconstrução de trânsito para outras afecções benignas ou mesmo carcinoma $a^{1,3,6,13,14}$.

A fibrose que se instala na evolução do fechamento espontâneo da fístula da anastomose cervical faz com que muitos doentes também evoluam com estenose. Isto ficou bem evidente nesta série, pois dos cinco pacientes que apresentaram estenose, quatro haviam apresentado previamente deiscência da mesma. Esta complicação faz com que retarde ainda mais o início da deglutição, com comprometimento da qualidade de vida na avaliação precoce, embora após a dilatação endoscópica, todos tenham apresentado deglutição satisfatória para sólidos e pastosos, entre o $50^{\circ}$ e $80^{\circ}$ dia de pós-operatório. Outras séries que utilizam este tipo de reconstrução apresentam resultados similares ${ }^{6,14,23}$.

Outra desvantagem da esofagocoloplastia é ser operação de maior complexidade, com necessidade de maior manipulação tecidual pela realização de várias anastomoses para reconstrução mais funcional do trato digestivo. A isto se soma o fato de todos os pacientes desta série já terem sido submetidos à operação prévia, o que exigiu maior tempo cirúrgico e anestésico, predispondo à maior instabilidade hemodinâmica no intra e no pós-operatório imediato. Estas alterações muitas vezes predispõem à complicações cardiovasculares ou mesmo pleuropulmonares, principalmente por se tratar de pacientes potencialmente comprometidos pela doença chagásica. Isto se refletiu também nesta casuística: $20 \%$ dos pacientes apresentaram complicações cardiovasculares, com evolução fatal em um deles e 30\% infecção pulmonar, mas com boa evolução com tratamento clínico. A frequência de $10 \%$ de óbito, embora não desprezível, é aceitável, por se tratar de pacientes que, na avaliação pré-operatória, já apresentavam alterações cardiovasculares, por miocardiopatia chagásica. Outros autores também têm demonstrado complicações sistêmicas quando da execução da esofagocoloplastia ${ }^{13,14,23}$.

Outra complicação que tem sido descrita, porém bastante incomum, é a necrose do colo interposto, devido às alterações anatômicas vasculares que podem ocorrer neste órgão, desde que não haja nenhum erro técnico ${ }^{9,10,23}$. Apesar de nenhum paciente desta série ter apresentado esta complicação, seria necessário realizar estudo com maior casuística para que se possa avaliar de maneira mais objetiva a sua real incidência.

Os poucos autores que preferem a transposição colônica em relação à gástrica, relatam que, apesar desta víscera apresentar boa vascularização com uma só anastomose para reconstrução do trânsito digestivo, tem a desvantagem de proporcionar maior potencial de aspiração do conteúdo gástrico, além de estenose cervical, proveniente do refluxo crônico, principalmente quando se consegue acompanhar os pacientes a longo prazo $^{9,17,22,23}$.

Dos 194 pacientes em que foi realizada a transposição gástrica para reconstrução de trânsito após a esofagectomia para megaesôfago grau III/IV, poucos deles referiram clínica de refluxo, evidenciando assim, a maior validade deste órgão para substituição esofágica ${ }^{2}$.

\section{CONCLUSÕES}

A reconstrução com o colo em pacientes submetidos à esofagectomia por megaesôfago avançado não deve ser a primeira opção de tratamento, tendo somente indicação quando for inviável a transposição gástrica, e ela deve ser considerada técnica cirúrgica com alta morbimortalidade em pacientes previamente gastrectomizados. 
Aquino JLB, Said MM, Maziero D, Pliveira JMP, Leando-Merhi VA. Esophageal reconstruction by esophagocoloplasty for megaesophagus end-stage in gastrectomized patients. ABCD Arq Bras Cir Dig 2009;22(4):197-200

ABSTRACT - Background - Esophagocoloplasty in advanced chagasic megaesophagus is seldom necessary. But, when realized, brings with it a major incidence of complications. Aim - To verify the complications following esophagus reconstruction by esophagocoloplasty in patients with previous gastrectomy submitted to transmediastinal esophagectomy for chagasic megaesophagus end-stage. Methods - From July 1983 to April 2009,204 patients with chagasic megaesophagus stage III and IV were submitted to esophageal resection at the Surgery Department at the Hospital Celso Pierro Puc-Campinas, Brasil. Ninety-two patients had the procedure done by esophageal mucosectomy; 84 under-went a transhiatal esophagectomy and in 38, esophagectomies were done through a transthoracic approach. In 194 patients (95\%) the reconstruction was done by gastric interposition and in the 10 remaining patients (5\%) it was by transposition of the transverse colon. This procedure was recommend due to previous gastrectomy done in all patients. All of them were male with average age of 47.5 years old. Results - Seven patients (70\%) developed one or more complications, either systemic or local. Among the systemic complications, one patient had thrombophlebitis in the lower extremity, with good recovery; one had a myocardial infarction with fatal evolution; three patients $(30 \%)$ had pulmonary infection with good recovery. Amongst the local complications, four patients had cervical anastomotic leak with satisfactory recovery with conservative treatment. Five had cervical anastomotic stricture between the 35 th and the 63rd post-operative day, with good recovery after endoscopic anastomotic dilatations. From the eight patients followed during this period, six (75\%) had good deglutition for solids and/or semi-solids, and satisfied with the surgery since were able to return to their regular diet. Conclusions - Despite the fact that the esophagocoloplasty results in a good deglutition and quality of life, in short and medium terms, it must be considered a surgical technic with high morbidity in gastrectomized patients.

HEADINGS - Esophageal reconstruction. Chagasic megaesophagus. Esophagocoloplasty

\section{REFERÊNCIAS}

1. Aquino, JLB. Avaliação da anastomose esofagogástrica cervical com sutura mecânica em pacientes com megaesôfago avançado. Rev Col Bras Cir 2005; 3(2): 143-6.

2. Aquino JLB, Said MM, Maziero D et AL. Avaliação da esofagolocoloplastia na reconstrução de trânsito pós-esofagestomia por megaesôfago avançado em pacientes gastrectomizados. Ann Cong CBC 2009; 28:p.285.

3. Aquino JLB, Camargo JGT, Said MM et al. Avaliação da anastomose esofagogástrica cervical com sutura mecânica e manual em pacientes com megaesôfago avançado. Rev Col Bras Cir 2009; 36(1):019-023.

4. Aquino JBL, Morais SP, Muraro COM et al. Avaliação da fístula anastomótica na cirurgia do esôfago. In: Congresso Brasileiro de Cirurgia Digestiva, 1. São Paulo: Robe; 1993, 25-31.

5. Aquino JLB, Ishida $P$, Muraro $C O$ et al. Avaliação da sutura mecânica no esôfago cervical. In Congresso Brasileiro dos Cirurgiões, 20, Rio de Janeiro, 1993. Anais, p. 47.

6. Devaney EJ, Iannettoni MD, Orringer MB et al. Esophagectomy for achalasia: patient selection and clinical experience. Ann Throac Surg 2001; 72:854-8.

7. Glatz SM, Richardson JD. Esophagectomy for end-stage achalasia. J Gastrointes Surg 2007; 11(9):1143-7.

8. Gutiérrez AG, Oramas HP, Zambrana FR et al. Evaluación de los resultados de 230 esofagoplastias com segmentos pediculados de colon, yeyuno y estómago. Rev Cubana Cir 2001; 40: 457-61.

9. Hsu HS, Wang CY, Hsieh CC et al. Short-segment colon interposition for end-stage achalasia. Ann Thorac Surg 2003; 76:1706-10.

10. Maish MS, DeMeester SR. Indications and technique of colon and jejunal interpositions for esophageal disease. Sur Clin N Am 2005, 83: 505-514.

11. Martins P, Morais BB, Cunha-Melo JR. Postoperative complications in the treatment of chagasic megaesophagus. Int Surg 1993; 78:99-102.
12. Miller DL, Allen MS, Trastek VF et al. Esophageal resection for recurrent achalasia. Ann Thorac Surg 1995;60:922-6.

13. Orringer MB, Marshall B, Iannettoni MD. Transhiatal Esophagectomy for treatment of benign and malidnant esophageal disease. World J Surg 2001: 25, 196-203,

14. Orringer MB, Marshall B, Chang AC et al. Two thousand transhiatal esophagectomies: changing trends, lessons learned. Ann Surg 2007; 246: 363-374.

15. Orringer MB, Orringer JS. Esofagectomy: definitive treatment for esophageal neuromotor dysfunction. Ann Torac Sur 1982; 34: 237-48.

16. Orringer MB, Stirling MC. Esophageal resection for achalasia: indications and results. Ann Thorac Surg 1989; 47: 340-5.

17. Peters JH, Kauer WKH, Crookes PF et all. Esophageal resection with colon interposition for end-stage achalasia. Arch Surg 1995; 130: 632-6.

18. Rassi L. Resultados do tratamento do megaesôfago chagásico com uma variante de Heller. Rev Goiana Med 1965; 11:49-57.

19. Rassi L. Esofagocoloplastia retroesternal no tratamento cirúrgico do dólicomegaesôfago. Rev Goiana Med 1968; 14:111-112.

20. Rassi L. Cirurgia do megaesôfago: critério seletivo. Ann Cong Pan Am, XV, 1977: 157.

21. Rassi L. Critério seletivo na indicação da técnica cirúrgica para o megaesôfago chagásico. Rev Goiana Med 1979; 25:85-104.

22. Watson TJ, De Meester TR, Kauer WKH et al. Esophageal replacement for endstage benign esophageal disease. J Thorac Cardiovasc Surg 1998; 115:1241-9.

23. Young MM, Deschamps C, Trastek VF et al. Esophageal reconstruction for benign disease: early morbidity, mortality and functional results. Ann Thorac Surg 2000; 70:1651-5.
Fonte de financiamento: não há Conflito de interesse: não há Recebido para publicação:01/08/2009 Aceito para publicação: 14/10/2009 